\title{
Evidence for repetitive load in the trapezius muscle during a tapping
}

\section{task}

\section{Journal Article}

\section{Author(s):}

Tomatis, Laura; Müller, Christian; Nakaseko, Masaru; Läubli, Thomas

Publication date:

2012-08

Permanent link:

https://doi.org/10.3929/ethz-b-000042119

Rights / license:

In Copyright - Non-Commercial Use Permitted

Originally published in:

European Journal of Applied Physiology 112(8), https://doi.org/10.1007/s00421-011-2290-8 


\title{
Evidence for repetitive load in the trapezius muscle during a tapping task
}

\author{
L. Tomatis $\cdot$ C. Müller $\cdot$ M. Nakaseko $\cdot$ T. Läubli
}

Received: 3 June 2011 / Accepted: 13 December 2011 / Published online: 23 December 2011

(C) Springer-Verlag 2011

\begin{abstract}
Many studies describe the trapezius muscle activation pattern during repetitive key-tapping focusing on continuous activation. The objectives of this study were to determine whether the upper trapezius is phasically active during supported key tapping, whether this activity is cross-correlated with forearm muscle activity, and whether trapezius activity depends on key characteristic. Thirteen subjects ( $29.7 \pm 11.4$ years) were tested. Surface EMG of the finger's extensor and flexor and of the trapezius muscles, as well as the key on-off signal was recorded while the subject performed a 2-min session of key tapping at $4 \mathrm{~Hz}$. The linear envelopes obtained were cut into single tapping cycles extending from one onset to the next onset signal and subsequently time-normalized. Effect size between mean range and maximal standard deviation was calculated to determine as to whether a burst of trapezius muscle activation was present. Cross-correlation was used to determine the time-lag of the activity bursts between forearm and trapezius muscles. For each person the mean and standard deviation of the cross-correlations coefficient between forearm muscles and trapezius were determined. Results
\end{abstract}

Communicated by Arnold de Haan.

L. Tomatis $(\bowtie)$

Institute of Human Movement Sciences and Sport,

Swiss Federal Institute of Technology, Zurich, Switzerland

e-mail: tomatisl@ethz.ch

L. Tomatis $\cdot$ C. Müller $\cdot$ T. Läubli

Division of Public and Organizational Health, University

of Zurich and Swiss Federal Institute of Technology,

Zurich, Switzerland

M. Nakaseko

Graduate School of Health Care Sciences,

Jikei Institute, Osaka, Japan showed a burst of activation in the trapezius muscle during most of the tapping cycles. The calculated effect size was $\geq 0.5$ in $67 \%$ of the cases. Cross-correlation factors between forearm and trapezius muscle activity were between 0.75 and 0.98 for both extensor and flexor muscles. The crosscorrelated phasic trapezius activity did not depend on key characteristics. Trapezius muscle was dynamically active during key tapping; its activity was clearly correlated with forearm muscles' activity.

Keywords Trapezius myalgia $\cdot$ Key tapping $\cdot$ EMG . Phasic activity

\section{Introduction}

Musculoskeletal complaints in the neck and upper extremity, particularly trapezius myalgia, are common events in modern society. There is evidence for a possible causal relationship between computer work and musculoskeletal diseases in the neck and arm (Ming and Zaproudina 2003; Wahlstrom 2005; Gerr et al. 2006). Trapezius myalgia is mostly associated with static work in front of a computer with a fixed posture, stressful jobs, and insufficient rest (Madeleine 2010). It has been suggested that individuals with a poor computer working technique work with higher muscle activity in the forearm and shoulder (Lindegard et al. 2003). Wrist and arm postures, finger movements, speed of movements, and force applied while keying are examples of variables included in this construct (Kadefors and Läubli 2002; Wahlstrom 2005; Gerr et al. 2006).

Good ergonomic conditions, the time-spent working with computers, and the influence of input devices are the most important aspects regarding work-related musculoskeletal diseases in the upper body. Observing subjects 
working with keyboards, a previous investigation showed that absence or presence of neck pain could be predicted by assessing if a neck flexion greater than $20^{\circ}$ was present (Baker et al. 2008). A correct placement of the visual unit is therefore extremely important. A relationship between duration of computer use and prevalence of musculoskeletal problems has been previously reported (Ming et al. 2004; Bhanderi et al. 2008). Nevertheless, a recent review by Waersted et al. (2010) showed only limited epidemiological evidence for the association of computer work and some of the clinical diagnoses related to musculoskeletal complaints. Although it remains questionable as to whether computer work leads to clinical MSD diagnoses, a number of studies point out the interactions between neck and shoulder pain and trapezius as well as forearm muscle activation patterns: Subjects with more severe upper extremity symptoms apply more force while using the keyboard (Feuerstein et al. 1997). In addition, reduced intramuscular coordination between extensor and flexor arm muscles is present when using keys with high force characteristics (Tomatis et al. 2009). One of the functions of the trapezius muscle is the stabilization of the shoulder; hence it allows the stabilization of the arm. Recent findings suggest that pain-induced changes in trapezius activity also change the coordination of the wrist extensor and flexor muscles (Falla et al. 2004; Samani et al. 2011). Therefore, a dependency between forearm muscles and trapezius activation during key tapping is very plausible. We hypothesize that by applying higher forces or because of bad forearm muscle coordination (i.e., high co-contractions of agonist and antagonist muscles) while working with different key characteristics, higher muscle activation might be found in the trapezius muscle, since in computer operators pain in the forearm muscle is often accompanied by trapezius myalgia.

This study focuses on trapezius muscle load using input devices (keys with different force-displacement characteristics) and with supported forearm. In subjects with musculoskeletal diseases higher average trapezius activity and reduced rest time (prolonged periods without muscle relaxation) during work were already described (Vasseljen and Westgaard 1996; Hägg and Astrom 1997; Sandsjo et al. 2000; Thorn et al. 2007). Goudy and McLean (2006) stated that in computer workers pain-afflicted subjects differ from pain-free controls mainly in the amount of muscular rest time. The contribution of all upper limb joints, including the shoulder, to single-finger tapping has been investigated by Dennerlein et al. (2007) with motion analysis, showing that the shoulder contributes to a small extent to the tapping movement. As only the joint movement was recorded but not the muscle activity, we suggest that the muscle activation related to the tapping might be observable to a higher extent than the actual joint movement.We intended to identify phasic activity during tapping by assessing activity in the trapezius muscle during repetitive and fast tapping tasks. Specifically, the objectives were: (1) to determine whether the trapezius is phasically active during supported key tapping, (2) to determine if the trapezius activity depends on the forearm activity, and (3) to determine whether the strain intensity depends on the characteristics of the key.

\section{Methods}

Subjects

Thirteen right-handed subjects (seven women and six men) were included in the study, with the following anthropometric characteristics (mean $\pm \mathrm{SD}$ ): age $29.7 \pm 11.4$ years (ranging from 20 to 57 years) and height $171.8 \pm 9.7 \mathrm{~cm}$ (ranging from 155 to $187 \mathrm{~cm}$ ). All subjects worked at least $5 \mathrm{~h}$ per week at the computer. None of the subjects suffered from neck, shoulder, arm, or wrist pain.

The Ethics Committee of the ETH Zurich approved the study protocol, and informed consent to the procedure was obtained from all subjects.

The subjects were allowed to stop at any time in case of pain or fatigue.

\section{Experimental design}

The subjects sat with the right forearm supported on a table, the wrist sustained on a keyboard support, and the prone hand and fingers extended above the keyboard. The subjects had the possibility to adjust the chair to sit more comfortably.

The subjects were asked to depress the key with the index finger at a frequency of $4 \mathrm{~Hz}$ during $120 \mathrm{~s}$ while keeping the finger on the key. The pace was provided by audio signals. This tapping task was repeated once for each of the ten keys with different characteristics (Table 1) in random sequence (Tomatis et al. 2009) to avoid an order effect.

Table 1 Key force-displacement characteristics and labels

\begin{tabular}{|c|c|c|c|c|c|c|c|c|c|c|}
\hline Key name & $40 \mathrm{p}$ & $60 \mathrm{p}$ & $80 \mathrm{p}$ & $100 \mathrm{p}$ & $120 \mathrm{p}$ & $1 \mathrm{~mm}$ & $2 \mathrm{~mm}$ & $3 \mathrm{~mm}$ & $4 \mathrm{~mm}$ & $5 \mathrm{~mm}$ \\
\hline Make-force (N) & 0.39 & 0.59 & 0.78 & 0.98 & 1.18 & 0.59 & 0.59 & 0.59 & 0.59 & 0.59 \\
\hline Key displacement (mm) & 3 & 3 & 3 & 3 & 3 & 1 & 2 & 3 & 4 & 5 \\
\hline
\end{tabular}


The surface electromyogram (sEMG) of the finger extensor ( $m$. extensor digitorum) and flexor ( $m$. flexor digitorum) and of the trapezius ( $m$. trapezius descendens) muscle was recorded, as was the key on-off signal.

\section{Key characteristics}

The keys differed in their force-displacement characteristics: five keys had the same displacement $(3 \mathrm{~mm})$ but differing in forces, and the other five had the same force $(0.588 \mathrm{~N})$, but different displacements (Table 1).

\section{Electrodes}

Conventional surface bipolar $\mathrm{Ag} / \mathrm{AgCl}$ electrodes $(20 \mathrm{~mm}$ apart, pre-gelled, $9 \times 6 \mathrm{~mm}$ recording area, Medtronic, Switzerland) were used to record the sEMG signals.

Before applying the electrodes, the skin was shaved and prepared with a peeling paste. Bipolar electrodes were placed at a point $2 / 3$ of the distance from $\mathrm{C} 7$ and the acromion (Jensen et al. 1993). Both the extensor and flexor application points on the forearm were found by palpation. A reference electrode was placed on $\mathrm{C} 7$.

\section{Hardware}

An eight-channel pre-amplifier (GAIN = 100) and an eightchannel amplifier with manual adjustment for amplification (10-50), a 30-Hz high-pass filter, a 300-Hz low-pass filter and a 50-Hz notch filter (Signal and Information Processing Laboratory ETH Zurich, Switzerland) were used to record the measurements. No ECG artifacts were observed by visual inspection.

A 12-bit A/D card (NI PCI-6023E, National Instruments, Austin, Texas) was installed on a $1.10 \mathrm{GHz}$ personal computer (Windows XP) to sample and store the data. Data were stored at $2,048 \mathrm{~Hz}$ using custom software programmed with Matlab (Version 7.0.1, Mathworks, MA, USA).

\section{Data analysis}

The sEMG signals of the muscles extensor digitorum, flexor digitorum and trapezius were rectified and processed with a sixth-order Butterworth low-pass filter at $5 \mathrm{~Hz}$ to obtain linear envelopes, which provide information on the timing and duration of the burst, as well as details on muscle activation characteristics.

Using the onset signal of the key-tap, the linear envelopes obtained from each channel were cut from one key onset signal to the next one. To remove timing variability between the cycles, the envelope length was time normalized to 1,000 samples per cycle, using a re-sampling procedure.
For each condition and each subject, the limits for the outliers were defined as the 10th and 90th percentile of the amplitude range for every cycle. Outliers were excluded from the further analysis. For each session with a specific key characteristic approximately 400 until 500 cycles could be used. The normalized cycles were overlayed and the average activity level within a cycle was determined. At each point in time the mean and its standard deviation were calculated. The mean range over the tapping cycles was compared to its maximal standard deviation. The resulting quotient was used to describe the effect size of the observed trapezius activation (Leonhart 2004). The maximal amplitude of the averaged signal was used to characterize the phasic component of the trapezius activity. Therefore, the mean of the averaged signal was shifted to zero and the amplitude was normalized by setting the maximum to
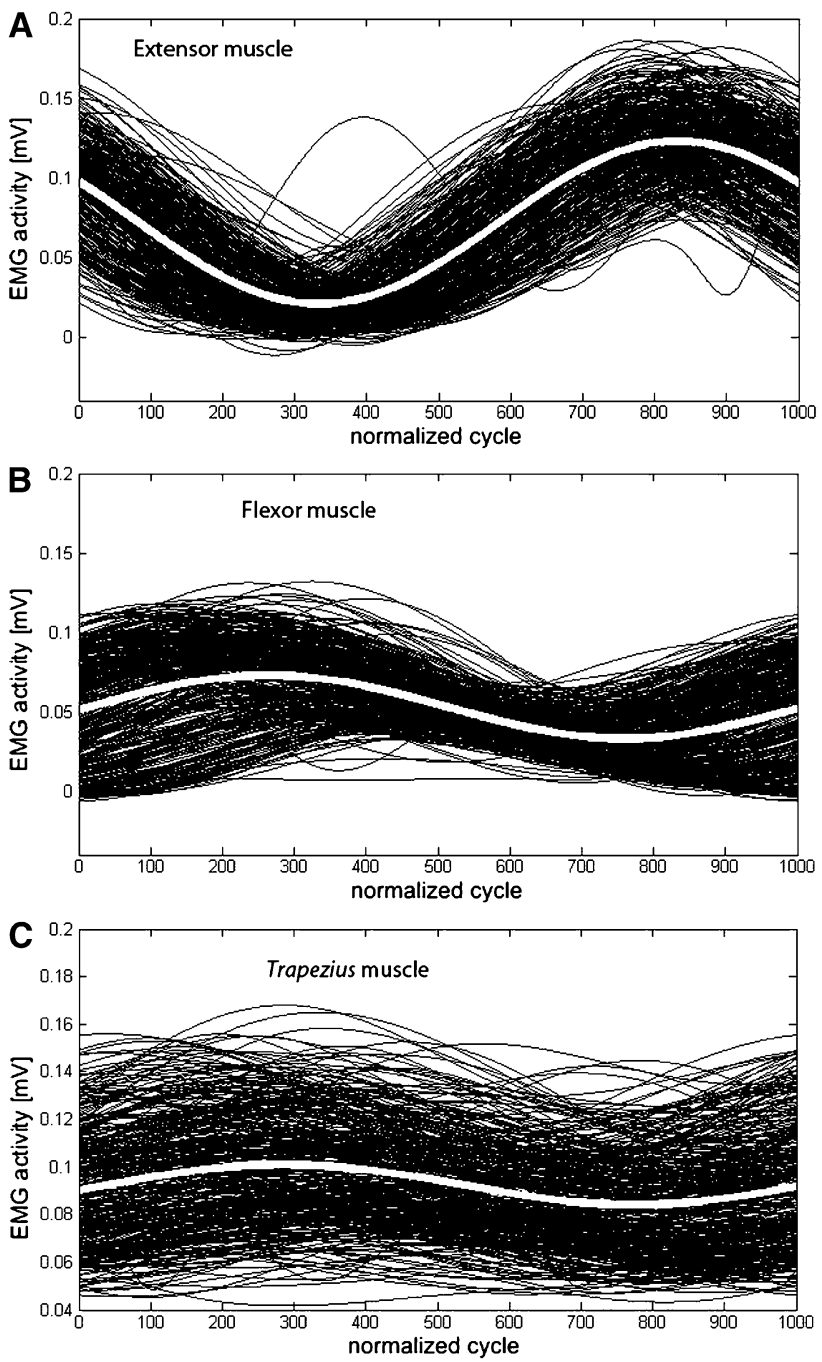

Fig. 1 a-c Depiction of the overlaid sEMG activities of the forearms and trapezius muscles during the tapping cycle of one subject. The thick white line represents the mean. The cycle period lasts approximately $250 \mathrm{~ms}$ divided into 1,000 normalized units 
Table 2 Effect size and significance of cross-correlation

\begin{tabular}{|c|c|c|c|c|c|c|c|c|c|c|}
\hline \multirow[t]{2}{*}{ Person } & \multicolumn{10}{|c|}{ Key name } \\
\hline & $40 \mathrm{p}$ & $60 \mathrm{p}$ & $80 \mathrm{p}$ & $100 \mathrm{p}$ & $120 \mathrm{p}$ & $1 \mathrm{~mm}$ & $2 \mathrm{~mm}$ & $3 \mathrm{~mm}$ & $4 \mathrm{~mm}$ & $5 \mathrm{~mm}$ \\
\hline 1 & $0.4^{*}$ & $2.86^{*}$ & $2.67 *$ & $3.03 *$ & $2.94 *$ & $2.99 *$ & $2.59^{*}$ & $2.17 *$ & $1.60 *$ & $1.64 *$ \\
\hline 2 & $0.74 *$ & $0.96 *$ & $0.81 *$ & $1.11^{*}$ & $0.66^{*}$ & $0.94 *$ & $0.64 *$ & $0.62 *$ & $0.96^{*}$ & $1.08 *$ \\
\hline 3 & $0.36^{*}$ & $0.50 *$ & $0.47 *$ & $0.71 *$ & $1.05^{*}$ & $0.66^{*}$ & $0.51 *$ & $0.94 *$ & $0.94 *$ & $0.13 *$ \\
\hline 4 & $0.16^{*}$ & $0.47 *$ & $0.61 *$ & $0.24 *$ & $0.85 *$ & $0.46^{*}$ & $0.47 *$ & $0.86^{*}$ & $0.44 *$ & $0.36^{*}$ \\
\hline 5 & $0.73 *$ & $0.33 *$ & $0.86 *$ & $0.52 *$ & $0.63 *$ & $0.60 *$ & $0.24 *$ & $0.43 *$ & $0.65 *$ & $0.81 *$ \\
\hline 6 & $0.46^{*}$ & $0.53 *$ & $1.24 *$ & $0.92 *$ & $1.60 *$ & $1.51 *$ & $0.36 *$ & $0.29 *$ & $2.13 *$ & $0.49 *$ \\
\hline 7 & $1.18^{*}$ & $0.28 *$ & $0.52 *$ & $1.29 *$ & $1.07 *$ & $0.30 *$ & $0.39 *$ & $0.35^{*}$ & $0.46^{*}$ & $0.91 *$ \\
\hline 8 & $1.56^{*}$ & $1.92 *$ & $2.28 *$ & $2.54 *$ & $1.88^{*}$ & $1.24 *$ & $1.13 *$ & $0.84 *$ & $1.20 *$ & $1.18 *$ \\
\hline 9 & $1.20 *$ & $0.53 *$ & $0.37 *$ & $1.52 *$ & $1.12 *$ & $0.97 *$ & $0.77 *$ & $0.93 *$ & $0.61 *$ & $1.38^{*}$ \\
\hline 10 & $0.46^{*}$ & $0.45^{*}$ & $0.25^{*}$ & $0.11 *$ & $0.64 *$ & $0.43^{*}$ & $0.44 *$ & $0.62 *$ & $0.29 *$ & $0.40 *$ \\
\hline 11 & $0.43^{*}$ & $0.27 *$ & $0.25 *$ & $0.65^{*}$ & $0.55^{*}$ & $0.41 *$ & $0.34 *$ & $0.53 *$ & $0.62 *$ & $0.46^{*}$ \\
\hline 12 & $0.34 *$ & $0.14 *$ & $0.64 *$ & $0.74 *$ & $1.34 *$ & $1.03 *$ & $0.44 *$ & $0.63 *$ & $0.45^{*}$ & $0.38^{*}$ \\
\hline 13 & Miss & $0.63^{*}$ & $1.28 *$ & $0.99^{*}$ & $1.29 *$ & $1.23 *$ & $0.74 *$ & $0.60 *$ & $0.82 *$ & $0.90 *$ \\
\hline
\end{tabular}

$* p<0.001 \%$

$100 \%$. Thus, the resulting signal ranges from approximately $-100 \%$ to a maximum of $100 \%$.

Statistical methods

Statistical analysis was performed using SAS (Version 9.1, SAS Institute Inc., NC, USA).

To check if the activity bursts of the forearm and trapezius muscles are time-correlated during the tapping cycle, cross-correlation was used.

Mixed models statistics (proc mixed) were used to calculate exact $p$ values and significances. The correlation coefficients determined by cross-correlation were regressed on the predictor key and order of key. Subjects were set as a random factor. Significance was assumed for $p \leq 0.05$.

\section{Results}

Objective 1: Phasic activation of trapezius muscle

Results showed a phasic activation of the trapezius muscle during the tapping cycle (Fig. 1). The calculated effect size was $\geq 0.5$ in $67 \%$ of the cases (graphs), where a case is defined as all repetitions for one key and one subject (Table 2).

Objective 2: Trapezius activity depends on forearm activity

The size of the correlation coefficient determined by the cross-correlation between forearm and trapezius muscle activity ranged between 0.75 and 0.98 (mean $0.93 \pm 0.05$ ) for both the extensor and flexor muscle.

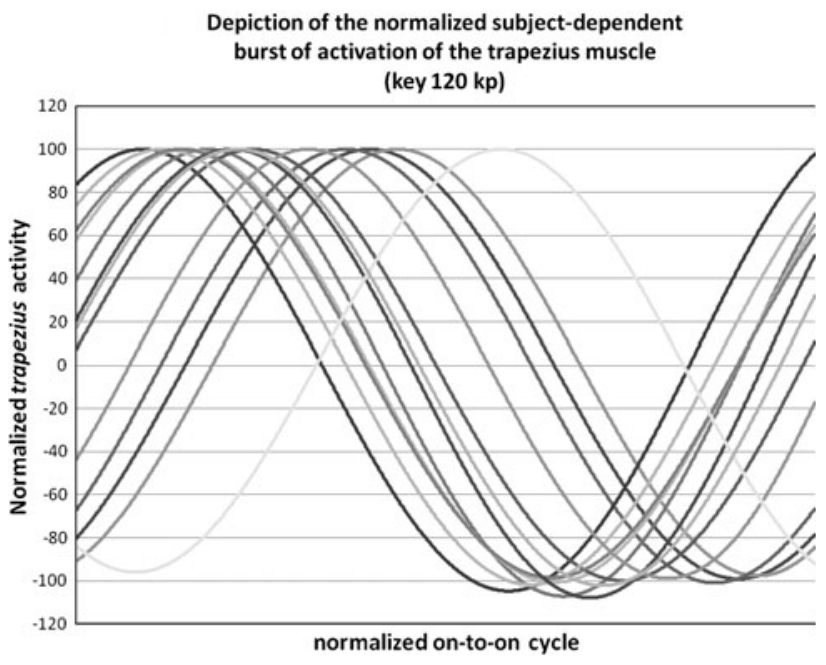

Fig. 2 Depiction of the burst of activation of all subjects during the tapping cycle obtained with the key characteristic $120 \mathrm{kp}$. Every cycle has been normalized for time and amplitude

Phasic activity of the trapezius muscle was nearly always detectable, but at significantly different time-points within the tapping cycle for the participating subjects (Fig. 2).

Objective 3: Dependency of trapezius activity to key characteristics

Mixed models statistics were used to examine the relationship between the timing of the trapezius activation and the different key characteristics. Trapezius activity did not 
Table 3 Results of the mixed models statistic used to calculate the influence of different subjects and keys on the correlation coefficient of the flexor and extensor muscles

\begin{tabular}{lrc}
\hline Variable & F & $p$ \\
\hline Flexor & & \\
Subject & 34.79 & $<0.01$ \\
Key & 1.71 & 0.10 \\
Order & 0.28 & 0.60 \\
Extensor & & \\
Subject & 37.03 & $<0.01$ \\
Key & 1.66 & 0.11 \\
Order & 0.53 & 0.47 \\
\hline
\end{tabular}

strongly depend on key characteristic neither for flexor muscle nor for extensor muscle (Table 3).

\section{Discussion}

Objective 1: Phasic activation of trapezius muscle

One of the aims of this study was to provide evidence for repetitive strain in the trapezius muscle during a tapping task. In $67 \%$ of the studied cases a burst of activation was detected with an effect size $\geq 0.5$.

Increased phasic trapezius EMG activity during fingeror key-tapping was shown. In 2000, Schnoz et al. found elevated dynamic and static trapezius muscle activity during finger tapping at different rates and trunk postures that were not only explainable by mechanical reasons such as maintenance of body posture. In addition, Zennaro et al. (2003) found continuous active motor units during $30 \mathrm{~min}$ of key tapping, supporting the Cinderella hypothesis (Hägg 1991).

To exclude a possible correlation with the movement of the upper arm, we chose a setup with supported forearm and supported wrist. Under those conditions, shoulder and arm are almost immobile: no movement was visible during the tapping task. Nevertheless, we detected repetitive activation of the trapezius muscle in correlation with activation of the forearm muscles. The observed activity shows a phasic pattern and is highly time-correlated with the key on-off signal. Many studies showed generally increased EMG values during key tapping, describing a more or less static EMG component, for which the source remained unclear (Zennaro et al. 2003; Leonard et al. 2010; Madeleine 2010). In the experiment conducted, the tapping was performed with only one finger at a given speed. Therefore, the phasic activation of the trapezius could easily be measured. Thinking about a more realistic work task, most workers would use a ten-finger system while working with a keyboard.
Using all fingers with some variation in speed should result in overlaying phasic activation patterns. Therefore, we hypothesize that a great part of the generally increased activity described in the aforementioned studies could be explained by the phasic activation as seen in our experiment. A possible explanation for the detected activity could be anticipatory postural adjustments (APAs) to stabilize the position of the segments of the body during movement (Massion 1992). Anticipatory postural adjustments are unconscious muscular activities preceding the voluntary movement aiming to prevent the changes in posture produced by the focal movement itself (Caronni and Cavallari 2009). In the year of 2009, Caronni and Cavallari conducted an experiment to investigate the role of APAs during index finger tapping. They showed that with the hand resting prone, APAs in trapezius muscle could not be observed. In contrast they found an inhibition of the trapezius prior to the finger tap in the prone position. As the subjects in the present study also performed the key tapping with the index finger in a prone position, APAs may not fully explain the activity found in our study.

Another explanation for the observed activity in the trapezius can be found in the literature describing motor learning:

Darainy and Ostry (2008) showed that following an arm movement learning task co-contraction of the shoulder still remained constant. In the initial phase of learning a new movement, very high activity can be observed in all muscles related to the movement, but co-contractions decrease with the learning progress (Thoroughman and Shadmehr 1999). According to Darainy and Ostry, these co-contractions do not disappear throughout the learning process but still form a central part of the means by which the nervous system regulates movement, also in highly skillful subjects. It seems that even though in our experimental setup the subjects were experienced keyboard users and performed the tapping task with supported forearm, the activation of the trapezius is not needed from a biomechanical point of view, but also cannot be avoided because it is part of the motor program controlling the movement.

Objective 2: Trapezius activity depends on forearm activity

The trapezius activity was found to be dependent on the forearm activity: cross-correlation ratios between forearm and trapezius activity were high for both extensor and flexor muscles.

Comparable results have been found by Schnoz et al. (2000) who showed that dynamic co-activity of the trapezius muscle occurs during computer mouse use and is timelinked with the mouse clicking. A recent publication of Samani et al. (2011) showed that artificially induced pain in 
the trapezius can lead to changes in the coordination of wrist flexor and extensor muscles. Thus, there seem to be strong interactions between the motor control of forearm and trapezius muscles, influencing each others' activation patterns. This hypothesis is supported by Alizadehkhaiyat et al. (2007), showing that a weak shoulder may predispose other joints, e.g., the elbow, to injuries caused by overuse.

This relationship might also explain the high inter-individual differences in the timing of the burst of trapezius activation that were found in the present study. Various individual conditions could influence the interactions between forearm and trapezius muscle activity: e.g., shoulder strength (Alizadehkhaiyat et al. 2007), shoulder pain (Samani et al. 2011), muscle imbalance in shoulder or forearm (Lewis et al. 2005) or level of forearm muscle coordination (Tomatis et al. 2009). These differences could explain why some subjects may be predisposed to easier MSD development caused by the mechanisms described by Sjogaard and Sogaard (1998).

\section{Objective 3: Dependency of trapezius activity to key characteristics}

No significant relationship between key characteristic and phasic trapezius activity was observed.

During the recent years, computer work time has increased (Dolton and Pelkonen 2004). Already in 2002, Kadefors and Läubli estimated that more than half of the population in Europe was using a computer at work. Extended periods of time are spent using input devices and, if some keyboards induce more muscular activity, the risk of MSD development could increase. To be able to reduce possible risk factors, the effect of different key characteristics on the trapezius activity is of great interest.

As shown in Table 3, our experiments could not show any relationships between phasic trapezius activity and key characteristics. Therefore, within our experimental conditions, a different keyboard does not seem to influence phasic trapezius activity. It has to be taken into consideration, that we only analyzed the time component of the muscle activity. There might be some changes in trapezius EMG amplitude induced by the different key characteristics. Further analysis should be made to provide more information about possible differences in EMG amplitude.

\section{Limitations}

There might be some concerns about the subject's body position, as it was not fully controlled. As aforementioned, a comparable activation pattern of the trapezius muscle was found in most of the subjects, leading to the conclusion that the observed activity is mainly caused by the tapping process and not by posture. Nevertheless a possible influence of the posture on the trapezius activation and time shift cannot be fully excluded and has to be considered as a limitation of the study.

Cross-correlations were used to assess the dependency between trapezius and forearm muscle activity. This procedure provides only a linear relationship between the timing of the measured EMG activation patterns. Even though the correlation parameter were very high $(0.93 \pm 0.05)$, the effect might be overestimated. Principal component analysis (PCA) would be an alternative to gain more detailed information about the dependencies of muscle activation timing and could be used in future studies.

\section{Conclusion}

Our experiments showed phasic trapezius activation during a tapping task with supported wrist and forearm. This trapezius activity is highly correlated with the finger flexor and extensor activation. The causes for this activity and whether it could be seen as a primary cause for developing pain should be further investigated.

\section{References}

Alizadehkhaiyat O, Fisher AC et al (2007) Strength and fatigability of selected muscles in upper limb: assessing muscle imbalance relevant to tennis elbow. J Electromyogr Kinesiol 17(4):428-436

Baker NA, Sussman NB et al (2008) Discriminating between individuals with and without musculoskeletal disorders of the upper extremity by means of items related to computer keyboard use. J Occup Rehabil 18(2):157-165

Bhanderi DJ, Choudhary S et al (2008) A community-based study of asthenopia in computer operators. Indian J Ophthalmol 56(1):5155

Caronni A, Cavallari P (2009) Anticipatory postural adjustments stabilise the whole upper-limb prior to a gentle index finger tap. Exp Brain Res 194(1):59-66

Darainy M, Ostry DJ (2008) Muscle cocontraction following dynamics learning. Exp Brain Res 190(2):153-163

Dennerlein JT, Kingma I et al (2007) The contribution of the wrist, elbow and shoulder joints to single-finger tapping. J Biomech 40(13):3013-3022

Dolton P, Pelkonen P (2004) Impact of computer use, computer skills and computer use intensity: evidence from workplace ERS 2004. CEEDP0081, Centre for Economic Performance

Falla D, Bilenkij G et al (2004) Patients with chronic neck pain demonstrate altered patterns of muscle activation during performance of a functional upper limb task. Spine 29(13):1436-1440

Feuerstein M, Armstrong T et al (1997) Computer keyboard force and upper extremity symptoms. J Occup Environ Med 39(12):11441153

Gerr F, Monteilh CP et al (2006) Keyboard use and musculoskeletal outcomes among computer users. J Occup Rehabil 16(3):265277

Goudy N, Mclean L (2006) Using myoelectric signal parameters to distinguish between computer workers with and without trapezius myalgia. Eur J Appl Physiol 97(2):196-209 
Hägg GM (1991) Lack of relation between maximal force capacity and muscle disorders caused by low level static loads: a new explanation model. 11th Congress of the International Ergonomics Association, Taylor and Francis, Paris

Hägg GM, Astrom A (1997) Load pattern and pressure pain threshold in the upper trapezius muscle and psychosocial factors in medical secretaries with and without shoulder/neck disorders. Int Arch Occup Environ Health 69(6):423-432

Jensen C, Nilsen K et al (1993) Trapezius muscle load as a risk indicator for occupational shoulder-neck complaints. Int Arch Occup Environ Health 64(6):415-423

Kadefors R, Läubli T (2002) Muscular disorders in computer users: introduction. Int J Ind Ergonom 30:203-210

Leonard JH, Kok KS et al (2010) Prolonged writing task: comparison of electromyographic analysis of upper trapezius muscle in subjects with or without neck pain. Clin Ter 161(1):29-33

Leonhart R (2004) Estimating effect sizes in clinical trials. Rehabilitation (Stuttg) 43(4):241-246

Lewis JS, Green A et al (2005) Subacromial impingement syndrome: the role of posture and muscle imbalance. J Shoulder Elbow Surg 14(4):385-392

Lindegard A, Wahlstrom J et al (2003) The impact of working technique on physical loads : an exposure profile among newspaper editors. Ergonomics 46(6):598-615

Madeleine P (2010) On functional motor adaptations: from the quantification of motor strategies to the prevention of musculoskeletal disorders in the neck-shoulder region. Acta Physiol 199:1-46

Massion J (1992) Movement, posture and equilibrium: interaction and coordination. Prog Neurobiol 38:35-56

Ming Z, Zaproudina N (2003) Computer use related upper limb musculoskeletal (ComRULM) disorders. Pathophysiology 9(3): $155-160$

Ming Z, Närhi M et al (2004) Neck and shoulder pain related to computer use. Pathophysiology 11(1):51-56
Samani A, Fernandez-Carnero J et al (2011) Interactive effects of acute experimental pain in trapezius and sored wrist extensor on the electromyography of the forearm muscles during computer work. Appl Ergon 42(5):735-740

Sandsjo L, Melin B et al (2000) Trapezius muscle activity, neck and shoulder pain, and subjective experiences during monotonous work in women. Eur J Appl Physiol 83(2-3):235-238

Schnoz M, Laubli T et al (2000) Co-activity of the trapezius and upper arm muscles with finger tapping at different rates and trunk postures. Eur J Appl Physiol 83(2-3):207-214

Sjogaard G, Sogaard K (1998) Muscle injury in repetitive motion disorders. Clin Orthop Relat Res 351:21-31

Thorn S, Sogaard K et al (2007) Trapezius muscle rest time during standardised computer work: a comparison of female computer users with and without self-reported neck/shoulder complaints. J Electromyogr Kinesiol 17(4):420-427

Thoroughman KA, Shadmehr R (1999) Electromyographic correlates of learning an internal model of reaching movements. J Neurosci 19(19):8573-8588

Tomatis L, Nakaseko M et al (2009) Co-activation and maximal EMG activity of forearm muscles during key tapping. Int J Ind Ergonom 39(5):749-755

Vasseljen O Jr, Westgaard RH (1996) Can stress-related shoulder and neck pain develop independently of muscle activity? Pain 64(2):221-230

Waersted M, Hanvold TN et al (2010) Computer work and musculoskeletal disorders of the neck and upper extremity: A systematic review. BMC Musculoskelet Disord 11:79-94

Wahlstrom J (2005) Ergonomics, musculoskeletal disorders and computer work. Occup Med (Lond) 55(3):168-176

Zennaro D, Läubli T et al (2003) Continuous, intermitted and sporadic motor unit activity in the trapezius muscle during prolonged computer work. J Electromyogr Kines 13:113-124 